\title{
EVOLUCION EXTRA HOSPITALARIA EN LOS PRIMEROS 12 MESES DE VIDA DE LOS PREMATUROS INGRESADOS AL CENTRO DE PREMATUROS DEL HOSP. L. CALVO MACKENNA. 1956-1957
}

Drs. JORGE HOWARD, INES EBENSPERGER, CLARA ROMAN, PATRICIA OLIVOS, PEDRO COFRE y Sra, ROSENDA SOTO.

Cátedra de Pediatría del Prof. Dr. A. Arizcia. Centro de Prematuros Hosp. L. Calro Mackenna.

Esta publicación tiene como objetivo analizar la evolución seguida por los prematuros egresados del Centro de Prematuros, hasta cumplir un año, y de este mido satisfacer una interrogante en el sentido de justificar la mantención da un Servicio, en el que el promedio de días de hospitalización de cada prematuro es riayor, y con un costo diario también más elevado.

Hemos analizado el desarrollo ponderal, morbilidad, mortalidad y la infiuencia que en estos rubros tuvieran las condiciones económico-sociales del ambiente far miliar en que se desarrollaron los prematuros después de su salida del Centro.

\section{RESULTADOS}

En el cuadro $N^{0} 1$ se aprecia que la mortalidad total del Centro de Prematuros del Hospital Luis Calvo Mackenna en el año 1956 , fué de $29,3 \%$ y en 1957 un $22,7 \%$.

\section{CUADRO No 1}

INGRESOS, FGRESOS $Y$ MORTALTDAD DE LOS PREMATUROS INGRESADOS AL CHNMRO DE PREMATD.

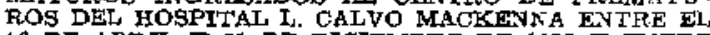
16 DE ABRE $Y$ T1 DE DICIEMBRE DE 1956 F ENTRE FI $1 \% \mathrm{DE}$ ENERO $\mathrm{Y}$ 3I DE DICIEMBRE $195 \%$. Santiago - Chlle

\begin{tabular}{|c|c|c|c|c|c|}
\hline \multirow[t]{2}{*}{ Años } & \multirow{2}{*}{$\begin{array}{l}\text { Ingresos } \\
\text { Totales }\end{array}$} & \multicolumn{2}{|c|}{ Egresos } & \multicolumn{2}{|c|}{ Mortalldad } \\
\hline & & $\mathrm{N}^{9}$ & $\%$ & Nọ & $\%$ \\
\hline $\begin{array}{l}1956 \\
1957\end{array}$ & $\begin{array}{l}310 \\
431\end{array}$ & $\begin{array}{l}219 \\
333\end{array}$ & $\begin{array}{c}70,6 \\
77,3\end{array}$ & $\begin{array}{l}91 \\
98\end{array}$ & $\begin{array}{l}293, \\
22,7\end{array}$ \\
\hline 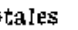 & 741 & 552 & 74,5 & 189 & 25,5 \\
\hline
\end{tabular}

EI análisis de la mortalidad por grupos de peso se encuentra desglosada en el cuadro No 2. Así tenemos que en 1956 el grupo de prematuros de $1.000 \mathrm{~g}$ o menos tuvo una mortalidad $94,7 \%$; para los niños entre 1.001 - $1.500 \mathrm{~g}$ un $46,5 \%$; para los de $1.501-2.000 \mathrm{~g}$, un $16,7 \%$ y para el grupo de más de 2 kI fué de $23.1 \%$.
Los cuadros 1 y 2 indican que las cifras de mortalidad dentro del Centro de Prematuros se pueden comparar con la de Centros de Prematuros de organización semejante $\mathrm{y}$ de diferentes países.

\section{CUADRO No 2}

MORTALIDAD (\%) DE LOS PREMATUROS INGRESADOS AL CENTRO DE PREMATUROS DEL HOSPITAL LUIS CALYO MACKENHA BNTRE BH 16 ABRIL AL 31 DE DLCLPATBEE 1956 Y ENNTRE EL 19 ENERO AL 31 DE DICIFMARE DE 1957. Santiago - Chile.

\begin{tabular}{|c|c|c|c|c|c|}
\hline Аี̆̈0, & $\begin{array}{l}\text { Mortalid } \\
\text { Grupo A } \\
-1000 \mathrm{~g}\end{array}$ & $\begin{array}{c}\operatorname{ad}\left(\frac{\pi}{3}\right) \\
B \\
1001-1500\end{array}$ & $\frac{C}{1501-2000}$ & ${ }_{2001-2500}^{D}$ & Total \\
\hline $\begin{array}{l}1956 \\
1957\end{array}$ & $\begin{array}{r}94,7 \text { \% } \\
100 \text { 总 }\end{array}$ & $\begin{array}{l}46,5 \text { \% } \\
32,4 \%\end{array}$ & $\begin{array}{l}16.7 \% \\
15,6 \%\end{array}$ & $\begin{array}{l}23,1 \text { w } \\
12,5 \text { \% }\end{array}$ & $\begin{array}{l}29,3 \\
22,7\end{array}$ \\
\hline
\end{tabular}

A título comparativo se muestra la mortalidad del año 1957 que indica una baja en la mortalidad, la que seguramente esta condicionada por un mejor conocimiento y tratamiento de las enfermedades propias del prematuro, como también y en forma especial a las mejores técnicas de enfermería aplicadas al cuidado de estos niños.

\section{CUADRO NO 3}

INGRESOS $Y$ MORTALIDAD DE LOS FREMATUROS CON EESO DF NACIMIENTO LNFERIOR A $1000 \mathrm{~N}$. HOS. PITALIZADOS EK EL CENTRO DE PREMATUROS PITALIZADOS EN ALRIL AL 31 DE DICIEMBRE DE 1956 Y ENTRE DU 19 DE ENERO AL 31 DE DICLEMBRE DE 1957.

Hospital Luls Calvo Maekenne - Santiago, Chile.

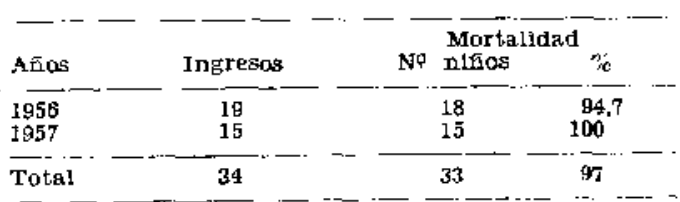

Del análisis de estos cuadros se desprende que en el cuadro $\mathrm{N}$ o 3 correspondiente a prematuros con peso de nacimien to inferior a 1.000 gramos, el número de niños que ingresan con este peso es muy pequeño y tenemos una mortalidad prácticamente del $100 \%$. 
CUADRO No 4

INGRESOS Y MORTALIDAD DE PREMATUROS CON PESO DE NACTMIENTO ENTRE $1001-1500 \mathrm{~K}$. HOSPITALIZACOS EN EL CENTTO DE PRGMATURÓS ENTPE EL 16 DE ABRIL AL 31 DB DICLEAMBRE 1956 Y ENTRE EL IO DE ENERO AL 31 DE DICIEMBRE DE 1957. Hospital Luls Calvo Mackenna - Sentlago, Chile.

\begin{tabular}{lccc}
\hline Afog & Ingresis & \multicolumn{2}{c}{ Motalidad nofinos } \\
\hline 1956 & 73 & 34 & 46,5 \\
1957 & 117 & 38 & 32,4 \\
\hline Total & 190 & 72 & 37,8 \\
\hline
\end{tabular}

Los grupos $B$ y C (Cuadros 4 y 5 ) tienen un mayor número de niños hospitalizados y los índices de mortalidad de estos prematuros son sensiblemente iguales a las publicadas por planteles de prematuros de otros paises.

\section{CUADRO NQ 5}

INGRRGOS $Y$ MORTALIDAD DE LOS PREMATUROS CON PDSO DE NACIMLENTO ENTRE 1501-2000 \& HOSPITALIZADOS EN KL CENTRO DE PREMATUROS ENTRE FL 16 AERIL AL 31 DE DICIEMBRE DE 1956 Y ENTRE EL 19 ENWRO AL 3I DE DICIFMBRE DE 1957. Fospltal Luls Calvo Mackenna - Santingo, Chlie.

\begin{tabular}{|c|c|c|c|c|}
\hline \multirow{2}{*}{$A \bar{n} 08$} & \multirow{2}{*}{ Ingresos } & \multicolumn{3}{|c|}{ Mortallded } \\
\hline & & NT & ninos & \\
\hline $\begin{array}{l}1850 \\
1857\end{array}$ & $\begin{array}{l}179 \\
293\end{array}$ & & $\begin{array}{l}30 \\
38\end{array}$ & $\begin{array}{l}16,7 \\
15,6\end{array}$ \\
\hline Total & 422 & & 68 & 18.1 \\
\hline
\end{tabular}

En el grupo D (Cuadro $\mathrm{N}^{\circ}$ 6) constituídos por niños con peso de nacimiento superior a $2.000 \mathrm{~g}$ el número de ingresos es menor $y$ el porcentaje de mortalidad mayor que la presentada por otras estadísticas, lo que estaría condicionado al hecho de permitir el ingreso sólo de aquellos niños que presentan alguna entidad mórbida.

\section{CUADRO N? 6}

INGRESOS $Y$ MORTALIDAD DE LOS PREMATUROS CON PESO DE NACIMIENTO ENTRE $2001-2500 \mathrm{~g}$. HOSPITALIZADOS EN EL CENTRO DE PREMATUROS ENTRE EL 16 DE ABRIL AL 31 DE DICIEMBRE DE $1956 \mathrm{Y}$ ENTRE BI 19 DE ENERO AL 31 DE DICIFMBRE DE 1957 .

Hospital Luis Calvo Mackenna. Santlago - Chlle.

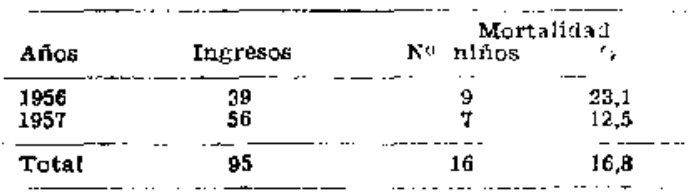

Producido el egreso desde el Centro de Prematuros, los niños fueron controlados en policlínica adjunta al Centro.
CUADRO N⿳ 7

CONTROL HASTA HE ANO DE BDAD DE LOS 219 PREMATUROS FGRESADOS DEL CENTRO DE PREMATUROS DEL HOSPITAL LUIS CALTO MACKENNA DURANTE KE, ANO 1956 . santlago - Chlle.

\begin{tabular}{|c|c|c|}
\hline & NS & $\%$ \\
\hline $\begin{array}{l}\text { Control en Pollclinica o en domicllio al } \\
\text { cumplir } 1 \text { año o más }\end{array}$ & 144 & 65,8 \\
\hline $\begin{array}{l}\text { Sin control médico del Servicto, pero } \\
\text { con informe conocldo de sobrevida al } \\
\text { año }\end{array}$ & 35 & 15,9 \\
\hline $\begin{array}{l}\text { Fallecldos en el hogar antes de cum- } \\
\text { plir } 1 \text { año de vida }\end{array}$ & 24 & 10,9 \\
\hline Total con evoluclon conocida & 203 & 82,6 \\
\hline Ignorados & 18 & 7.4 \\
\hline
\end{tabular}

De los 219 niños egresados se controló el $65,8 \%$ (144 niños) hasta cumplido el año de vida. No todos concurrieron a la citación periódica que se realiza en la policlínica, de tal modo que el conocimiento de su estado de salud fué conocido por el examen practicado al año de edad $y$ en el dominio de estos niños.

El rubro sin control médico del Servicio estuvo constituído por un grupo de niños que una vez registrado su egreso del hospital, siguió control con médico particular, o en otras Instituciones de Previsión o fueron controlados en Provincias.

De estos niños se obtuvo datos clínicos a través de testimonios pedidos verbalmente o por escrito, en los que se solicitaron informes sobre desarrollo pondo-estatural, estático-dinámico y sobre enfermedades intercurrentes tenidas durante este primer año de vida. Este grupo constituyo el $15,9 \%$ ( 35 niños).

Del total de niños egresados del Centro de Prematuros fallecieron $24(10,9 \%)$ en su hogar antes de cumplir el año de edad.

Conviene destacar que el número total de niños de Ios cuales tuvimos conocimiento fué del $92,6 \%$ (203) cifra muy aceptable y que refleja el esfuerzo de la enfermera sanitaria que mantiene un constante contacto con la familia de nuestros prematuros egresados.

De este modo escaparon a nuestro control solo 16 niños $(7,4 \%)$.

Del análisis del cuadro No 8 se desprende que la totalidad de los prematuros fallecidos en el primer, mes, Io hicieron durante su permanencia en el Centro de Prematuros. En cambio, de los falle- 
CUADRO NQ $\mathrm{B}$

MORTALIDAD DEL PRDMER ANO DF YIDA DE LOS PREMATUROS INGRESADOS Y CONTROLADOS DE EN POLICLINICO DEL CRNTRO DE PREMATUROS DEI HOSPITAL LUIS CALVO MACKENNA DDRANTE EL ANO 1956 .

Santiago - Cbile.

\begin{tabular}{|c|c|c|c|c|}
\hline & $\begin{array}{l}\text { Mortalldad } \\
\text { neonstai }\end{array}$ & & $\begin{array}{l}\text { Mortalldad de } \\
1-12 \text { ms. }\end{array}$ & $\begin{array}{l}\text { Mortalidad del } \\
\text { 1.er axdo }\end{array}$ \\
\hline $\begin{array}{l}\text { No } \\
\text { ros } \\
\text { el }\end{array}$ & $\begin{array}{l}\text { prematu- } \\
\text { fallecidos } \\
\text { l.er mes }\end{array}$ & Din & $\begin{array}{l}\text { No nifios fr- } \\
\text { liecidos en- } \\
\text { tre } 1 \text { I } 12 \\
\text { meses. }\end{array}$ & $\begin{array}{l}\text { Total prema- } \\
\text { turos falle- } \\
\text { cldos durad- } \\
\text { te el ler } \\
\text { mes. }\end{array}$ \\
\hline
\end{tabular}

$\begin{array}{lllll}78 & 25,2 & 37 & 12,6 & 115\end{array}$

NOTA: La mortalidad neonatal se calculo sobre los 310 ingresos, is mortalldad de 1 a 12 meses $5 e$ cajculo sobre 294 nifios, ya que hay 16 niños cuyo destino desplxés del primer mes se desconoce.

cidos entre el $1^{\circ}$ y $12^{\circ}$ meses, 13 murieron cuando aún permanecian en el Centro y 24 en su domicilio después del egreso.

Para considerar un niño eutrófico o distrófico dado la variabilidad de datos que hay que considerar para formular este diagnóstico en forma precisa, optamos por el criterio simple de considerar su peso, partiendo del hecho que nuestros prematuros aumentan de peso $300 \mathrm{~g}$ el primer mes, $600 \mathrm{gr}$. mensuales hasta los 9 meses y 500 gr. mensuales hasta el año, lo que da un aumento de $6.300 \mathrm{gr}$. al año.

El peso ideal al año de vida lo tomamos del peso de nacimiento más $6.300 \mathrm{~kg}$. Todo niño que tuviera peso igual o superior a dicha suma se consideró eutrófico y por el contrario, distrófico a todo niño que tuviera un peso inferior a esta suma. Tuvimos 77 niños $(53,4 \%)$ eutróficos con peso promedio de $8.920 \mathrm{~kg}$ y 67 niños $(46,6 \%)$ de distróficos con peso promedio de $7.060 \mathrm{~kg}$, como se ve en el cuadro No 9 .

CUADRO NO 9

FSTADO DE LOS PREMATUROS CONTROLADOS AL CUMPLIR 1 ANO DE EDAD

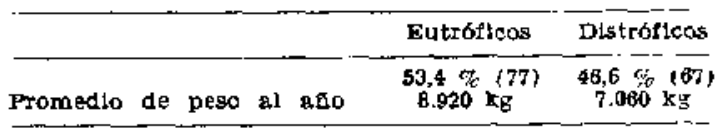

Del análisis del cuadro No 10 se desprende que el número de consultas desde un punto de vista cuantitativo fué semejante tanto para los niños eutróficos y distróficos, en cambio desde un punto de vista de la causal de consultas, como es lógico suponerlo, los distróficos concurrieron con mayor frecuencia a control por razones de enfermedad intercurrente
CUADRO Na 10

CONTROL EN POLICLINICO

\begin{tabular}{|c|c|c|}
\hline $\begin{array}{l}\text { Consultas por afio } \\
\text { Consultas n1no sano } \\
\text { Consultias nino enfermo }\end{array}$ & $\begin{array}{r}8,2 \% \\
58,5 \% \\
41,5 \%\end{array}$ & $\begin{array}{c}8 \% \\
41,2 \% \\
38,8 \%\end{array}$ \\
\hline $\begin{array}{l}\text { Enfermedaces: } \\
\text { Aparato resplratorio } \\
\text { Aparato dlsestivo } \\
\text { Otras enfermedades }\end{array}$ & $\begin{array}{ll}\mathbf{5 6} & \% \\
24 & \% \\
20 & \% \\
20 & \%\end{array}$ & $\begin{array}{l}40,5 \% \\
38,3 \% \\
21,2 \%\end{array}$ \\
\hline $\begin{array}{l}\text { Retardo psiquico } \\
\text { Paraplejla espástica }\end{array}$ & $0.2 \%(1)$ & $\begin{array}{l}4.48(3) \\
0\end{array}$ \\
\hline
\end{tabular}

(58\%). Los eutróficos solo concurrieron en un $41 \%$ por enfermedad.

Analizando las enfermedades motivo de consulta, se aprecia que estas fueron principalmente del aparato respiratorio $y$ digestivo.

Conviene destacar la poca incidencia de daños cerebrales ya que solo tuvimos 4 niños, lo que da un $2,8 \%$, y que está lejos de la aseveración corriente, en el sentido que Ios prematuros con frecuencia muestran daños cerebrales en su evo lución posterior.

CUADRO NQ 11

BOSPITALIZACION

\begin{tabular}{|c|c|c|}
\hline & Eutroria & Distrofla \\
\hline Hospltalleaclon & $13 \%(10)$ & $35.8 \%(24)$ \\
\hline $\begin{array}{l}\text { Dlspepsia } \\
\text { Toxicosis } \\
\text { NeumonIas } \\
\text { Herntas } \\
\text { Otras: }\end{array}$ & $\begin{array}{l}5 \\
0 \\
2 \\
3 \\
0\end{array}$ & $\begin{array}{r}11 \\
2 \\
3 \\
5 \\
3\end{array}$ \\
\hline $\begin{array}{l}\text { Cardiopatia cong, } \\
\text { Tbe. primaria } \\
\text { Osteomlelltis }\end{array}$ & & $\begin{array}{l}1 \text { Cardiopatis cong } \\
1 \text { Tbc primaris } \\
1 \text { Osteomielits }\end{array}$ \\
\hline
\end{tabular}

Al analizar el cuadro No 11 observamos que los niños distróficos debieron ser hospitalizados en mayor porcentaje $(35,8 \%)$ que los eutróficos $(13 \%)$. Las causas de hospitalización fueron especialmente trastornos nutritivos agudos.

CUADRO NO 12

DIAGNOBTICOS CLINICOS $Y$ EDAD DE MURRTE DE LOS PREMATUROS CONTROL ADOS WN FOLYCLINICOS DEL CENTRO DE PREMATUROS $Y$ FALLECIDOS EN DOMICILO ANTES DE CUMPLIR 1 ANO DE IRDAD

\begin{tabular}{|c|c|c|c|}
\hline Diagnóst1cog & No & $\%$ & Edad de muerte \\
\hline Neumonfas & 5 & 20.8 & 4 ms. $-5 \mathrm{~ms}-6$ ms. - \\
\hline $\begin{array}{l}\text { Dispepsles } \\
\text { Toxlcosis } \\
\text { Coqueluche } \\
\text { Cardiopatia } \\
\text { congendta }\end{array}$ & $\begin{array}{l}4 \\
3 \\
1\end{array}$ & $\begin{array}{r}16,6 \\
12,5 \\
4,2\end{array}$ & $\begin{array}{l}3 \mathrm{~ms}-5 \mathrm{~ms},(2)-6 \mathrm{~ms} \\
3 \mathrm{~ms} .-7 \mathrm{~ms}-9 \mathrm{~ms} . \\
9 \mathrm{~ms} .\end{array}$ \\
\hline $\begin{array}{l}\text { (nuerte brusca) } \\
\text { Calasas drnotadas }\end{array}$ & 10 & $\begin{array}{r}4,2 \\
41,7\end{array}$ & $\begin{array}{l}5 \mathrm{~ms} . \\
3 \mathrm{~ms} \text { (5) }-5 \text { ms. } \\
6 \mathrm{~ms} \text { (2) - } 7 \mathrm{~ms} \text { (2) }\end{array}$ \\
\hline Total & 24 & 100,0 & \\
\hline
\end{tabular}


De los 24 niños que fallecen después de ser dados de alta del Centro de Prematuros, 29,1\% (7) fallece de trastornos nutritjvo agudo y el $20,8 \%$ (5) de neumonía.

La causa de muerte no fué posible precisarla en el $41,7 \%$ porque el niño falleció en la casa sin atención médica o recurrió a Servicio de Posta con el niño agónico. El $70,8 \%$ de estos niños fallecieron en el primer semestre de la vida.

CUADRO NO 13

CONDICIONES ECONOMICO-SOCLALES DE LOS PREMATOROS CONTROLADOS AL CUMPLIR 1 AFO DE EDAD

\begin{tabular}{|c|c|c|c|}
\hline A. & económices & Butrofiss & Distroflas \\
\hline & $\begin{array}{l}\text { 1. Indigentes } \\
\text { 2. Asegurados } \\
\text { 3. Enpl. públlco a } \\
\text { particulir } \\
\text { 4. Comerclante o } \\
\text { Industrial } \\
\text { 5. Erofeslongl }\end{array}$ & $\begin{array}{c}0 \\
37,7 \%(29) \\
45,4 \%(35) \\
13,0 \%(10) \\
3.9 \%(3)\end{array}$ & $\begin{array}{l}14,9 \text { \% }(10) \\
71,6 \%(48) \\
5,9 \%(4) \\
5,8 \%(4) \\
1,5 \%(1)\end{array}$ \\
\hline B. 1 & $\begin{array}{l}\text { Hebltacion } \\
\text { 1. Buena } \\
\text { 2. Mala }\end{array}$ & $\begin{array}{lll}80,5 & \% & (62) \\
19,5 & \% & (15)\end{array}$ & $\begin{array}{lll}55 & (37) \\
45 & 8\end{array}$ \\
\hline
\end{tabular}

El reuulal to trinjmo que se conaldera para clasificar una habitación coma "buena" fué: con alcentarluado o fosa séptics con agua poteble con plso entablado

Relación persona - pleza

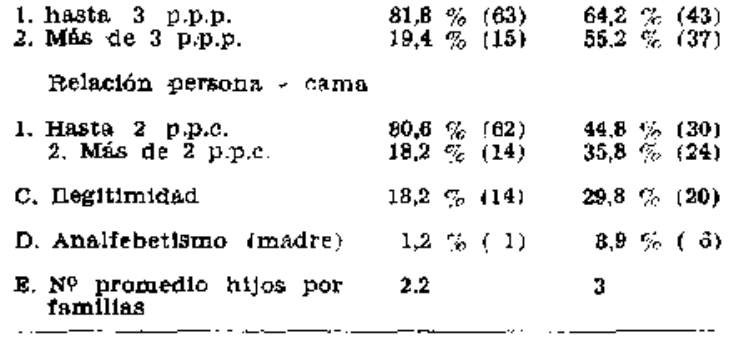

Se analizan las condiciones económico-sociales de los prematuros egresados del Centro. Se puede aprender como todos aquellos factores desfavorables se presentan en un mayor porcentaje entre los niños que al cumplir un año son distrófi$\cos$.

Podemos deducir de esto la importancia que estos factores ambientales tienen en el buen desarrollo de nuestros prematuros.

\section{RESUMEN}

Ss estudia la evolución de 219 prematuros que fueron dados de alta del Centro de Prematuros en un periodo de un año. De éstos se logró obtener datos concretos de $203(92,6 \%)$ hasta cumplir el año de edad, comprobándose que el $46,6 \%$ eran distróficos y en ellos las condiciones económico-sociales eran más desfavorables y tuvieron mayor número de hospitalizaciones. Los autores concluyen que el factor ambiental tiene gran importancia en el buen desarrollo del prematuro chileno.

\section{SUMMARY}

The authors studied a group of 219 prematures discharged from the Calvo Mackenna's Hospital Premature Center, and were able to obtain data on 203, $(92,6 \%)$ at one year of age. Of these $46,6 \%$ were malnourished, but in these the socio-economic conditions were significantly worse than in those that developed normaly. The authors stress the importance of the environment on the future development of the premature infant. 